\title{
Anti-Inflammatory Effect of the Isatis tinctoria L. Root Extract on Lipopolysaccharide-Induced Periodontitis in Rats
}

\author{
Sun Woo Lee ${ }^{1,2}$, Young Wook Cho ${ }^{2,3^{*}}$ \\ ${ }^{1}$ Korea International School, Jeju, Republic of Korea \\ ${ }^{2}$ Laboratory of Natural Pharmacology, BIOSTANDARD, Seoul, Republic of Korea \\ ${ }^{3}$ Department of Bioengineering, School of Engineering, Yon Sei University, Seoul, Republic of Korea \\ Email: *carpediemwj@gmail.com
}

How to cite this paper: Lee, S.W. and Cho, Y.W. (2019) Anti-Inflammatory Effect of the Isatis tinctoria L. Root Extract on Lipopolysaccharide-Induced Periodontitis in Rats. Journal of Biosciences and Medicines, 7, 81-95.

https://doi.org/10.4236/jbm.2019.79008

Received: July 26, 2019

Accepted: September 13, 2019

Published: September 16, 2019

Copyright $\odot 2019$ by author(s) and Scientific Research Publishing Inc. This work is licensed under the Creative Commons Attribution International License (CC BY 4.0). http://creativecommons.org/licenses/by/4.0/

\begin{abstract}
Objectives: Isatis tinctoria L., clary, is an herbal plant traditionally used in folk medicine for the treatment of various diseases and conditions. Although it has been primarily used as an antimicrobial and antifungal, there are data on traditional use of $I$. tinctoria as an agent against antiallergic, anti-thrombocytosis. The aim of the study was to examine the effect of the $I$. tinctoria root extract on the lipopolysaccharide (LPS)-induced periodontitis in rats on osteoclast associated bone resorptive activity, cell death including apoptosis, and inflammation in a rat of disease model of periodontitis. Materials and Methods: Periodontitis, acute or chronic inflammatory status in periodontal tissue in rats could be induced by repeated injections of LPS from Escherichia coli into the periodontal pocket area between the first and second right maxillary molars. Eighteen male rats were distributed among the following treatment groups: 1) I. tinctorial root extract (Antifect) $200 \mathrm{mg} / \mathrm{kg}$ body weight, 2) acetylsalicylic acid (ASA), $20 \mathrm{mg} / \mathrm{kg}$ body weight and 3) Phosphate buffered saline (PBS) treatment used as a control. After 15 days, maxilla, alveolar bone, molar teeth and associated periodontal tissues were harvested. Inflammatory alveolar bone resorption was analyzed by microcomputerized tomography ( $\mu \mathrm{CT}$ ) (microcomputer tomography). Tissues fixed with paraformaldehyde and formalin for 2 days, after that paraffin embedded histological sections were stained with haematoxylin and eosin (H/E) for the assessment of histopathological changes or tested to immunohistochemistry for detecting TRAP (tartrate resistant acid phosphatase) positive cells and caspase 3. Cell death and Apoptosis were analyzed in the periodontal tissues by tunnel assay. The inflammatory status was assessed by the measurements of proinflammatory cytokines interleukin-I $\beta$ (IL-I $\beta$ ), interleukin-6
\end{abstract}


(IL-6) and tumor necrosis factor- $\alpha$ (TNF- $\alpha$ ) of gingival tissues and descriptive analysis of histological sections of periodontal. Results: Treatment with Antifect, compared to the control group, significantly diminished the process of inflammation decreasing the levels of IL-I $\beta$, IL- 6 and TNF- $\alpha$, reducing the gingival tissue lesions and preserving bone alveolar resorption. Considerably a smaller number of inflammatory cells and a larger number of fibroblasts were noticed. Also, $\mu \mathrm{CT}$ analysis showed that only Antifect treated group reduced bone resorption and the number of TRAP-positive multinucleated cells (osteoclasts), also, significantly reduced the number of apoptotic cells in the gingival tissues and of osteocytes in the alveolar bone crest. Conclusion: Antifect manifested anti-inflammatory elect and reducing alveolar bone resorption in LPS-induced periodontitis suggest that it may have a role as a therapeutic agent in periodontal diseases.

\section{Keywords}

Isatis tinctoria, Antiinflammation, Antifect

\section{Introduction}

One major actor of the oral ecosystem is the dental plaque which develops naturally on hard and soft tissues of the mouth. This biofilm has a very complex organization that remains relatively stable with time despite regular environmental changes [1]. Periodontal disease is a chronic infective disease of the periodontal caused by periodontopathic bacteria accumulated on the tooth surface, characterized by destruction of the tooth supporting tissues including alveolar bone resorption [2] [3]. Subgingival Gram-negative organisms containing lipopolysaccharides (LPS) cause inflammation of the periodontal tissues inducing a local response, which implies a polymorphonuclear leukocyte infiltration, a production of reactive oxygen species (ROS) and inflammatory mediators such as cytokines and prostaglandins, amplification of lytic enzymes and activation of osteoclasts, edema and vascular dilatation [4] [5]. Recent investigations have shown that the gingival tissue from the inflamed area contains increased levels of some cytokines, primarily interleukin-1 $\beta$ (IL-I $\beta$ ), interleukin-6 (IL-6), and tumor necrosis factor- $\alpha$ (TNF- $\alpha$ ), implying that these cytokines mainly participate in the pathogenesis of periodontitis [6] [7] [8]. The culminating stadium of damaging effects of the produced cytokines and ROS is destruction of tooth-supported tissues including connective and mineralized tissue [9].

A remarkable number of studies have demonstrated the use of plants to be a new trend in the prevention and treatment of periodontal diseases with less adverse effects on humans. Some drugs for oral inflammations treatment can change oral microbiota and cause teeth discoloration [10] [11] [12]. In addition, clinical use of some phytochemicals is limited because of its poor absorption in the gastrointestinal tract, short plasma half-life and low bioavailability after oral 
administration [13]. Therefore, the search for natural products or phytochemicals is growing increasingly with prospects to become the acceptable alternatives or complementary agents for oral inflammations [14]. Bioactive phytochemicals from herbs such as phenolic acids, flavonoids, tannins, terpenoids, alkaloids etc. have been found to be particularly helpful in periodontitis due to their potent antimicrobial and anti-inflammatory activities [15]. Plant species from the genus Isatis, Brassicaceae family, especially Isatis tinctoria, are well known effectual agents whose active components could reduce inflammation, inhibit growth of bacteria and fungi and have beneficial effects on the prophylaxis [16] [17] [18].

Isatis tinctoria $\mathrm{L}$. has a long and well documented history as an indigo dye plant in temperate climates and as a medicinal herb. The original habitat of woad is probably in the grasslands of southeastern Russia; however, the plant was introduced early to Europe and Eastern Asia including Korea, China and Japan. Its medicinal properties have been extensively described in a number of Renaissance and Baroque herbals. Woad was recommended for the treatment of wounds, ulcers and tumours, haemorrhoids, snake bites and various inflammatory ailments [19]. Over the last decades, woad has been investigated both from a phytochemical and biological viewpoint. Extracts have been screened for antiviral, antifungal, antibacterial, cytoinhibitory and insecticidal activities, infusions were tested for antiallergic properties in humans [20] [21]. Inhibition of aggregation of thrombocytes has been reported [22].

Although there are data on traditional medicinal use of woad, there have been no studies concerning its inhibiting impact on the development of periodontitis on an animal model; in addition this is the first experimental research focusing on antiinflammatory properties of the I. tinctoria root extract (here in "Antifect"). In this pilot study, we investigated the effectiveness of the Antifect in suppressing the inflammation of the rats periodontal caused by LPS, by determining the levels of proinflammatory factors and histopathological analysis, as well as the chemical composition and antioxidant activity of the same extract.

\section{Materials and Methods}

\subsection{Experimental Design}

All animal experiments were conducted after obtaining the approval of the Seoul National University Institute of Laboratory Animal Resources and Use Committee. Ten weeks old $(247.5 \pm 54.16 \mathrm{~g})$, were kept in wire cages, in a room with proper, controlled conditions of temperature $\left(21^{\circ} \mathrm{C}-23^{\circ} \mathrm{C}\right)$ and humidity $(65 \%$ $70 \%)$ with $12 \mathrm{~h}$ light/dark cycle. The animals were fed with milled pellets, and had free access to water during the experiment. Lipopolysaccharide, derived from Escherichia coli (055:B5) and diluted in a sterile saline solution, was used for induction of periodontitis. One microliter of LPS solution $(10 \mu \mathrm{g} / \mu \mathrm{L})$ was slowly injected into interdental papilla between the first and second right maxillary molar [23], two times for fifteen days, using a Hamilton microsyringe. Rats were randomly divided into five experimental groups with six animals in each: 
(I) injected with saline, treated with vehicle (distilled water); (II) injected with saline, treated with Antifect; (III) injected with LPS, treated with vehicle; (IV) injected with LPS, treated with Antifect; (V) injected with LPS, treated with ASA. Groups I and II received the same volume $(1 \mu \mathrm{L})$ of sterile saline injection in the same location of rat maxilla. Groups II and IV received Antifect dissolved in water which was administered two times daily by oral gavage $(200 \mathrm{mg} / \mathrm{kg}$ body weight). Compounds were administered daily during 15 days by oral intubation beginning $24 \mathrm{~h}$ after the start of local PBS and LPS injections in the protocol for induction of experimental periodontal disease. Animals were euthanized by cervical dislocation under inhalation anesthesia and the hemi-maxillae were carefully dissected. Gingival soft tissue (approximately $1.5 \mathrm{~mm}$ in the frontal plane $\times 3 \mathrm{~mm}$ in the sagittal plane) adjacent to the upper first molars were carefully dissected from 5 samples from each experimental condition, immediately snap-frozen in liquid nitrogen and subsequently stored at $-80 \mathrm{C}$ until the moment of total protein extraction. The other 5 samples from each experimental condition were subjected to $10 \%$ buffered formalin fixation and subsequent histological processing for paraffin embedding (as described below) and used in histological and immunohistochemical analysis.

\subsection{Plant Material and Extraction}

Fresh and dried roots of woad (Isatis tinctoria L.) were provided in July 2018 by Dr. Ki Hyun Ryoo, Seoul, Republic of Korea, kept for medicinal use. Extracts for screening were prepared as follows: Dried roots were ground $(<0.7 \mathrm{~mm})$. An aliquot $(100 \mathrm{~g})$ was extracted at room temperature with $80 \%$ of $4: 6 \mathrm{v} / \mathrm{v}$ ratio of $\mathrm{CH}_{2} \mathrm{Cl}_{2}$ and $\mathrm{EtOH}(1000 \mathrm{ml})$ in and ultrasonic bath for 24 hours. Supercritical fluid extraction (SFE) was carried out with a Dionex 703 with 703M Modifier Module (Salt Lake City, USA). Removal of residual solvent was in a rotary vacuum evaporator at $40^{\circ} \mathrm{C}$. The average yield of extraction was $23.17 \% \pm 7.34 \%$.

\subsection{Microcomputer Tomography}

All hemi-maxillae (with and without soft tissues) were scanned on a microcomputer tomograph (Skyscan 1176, SkyScan Aartselaar, Belgium) using $18 \mu \mathrm{m}$ slices. Digital radiographic images of each sample were reconstructed into a three dimensional model and a standardized gray scale value was set to distinguish mineralized from non-mineralized tissues. These three dimensional images were re-oriented on the sagittal, coronal and transversal planes in a standardized manner using anatomical landmarks on molar teeth. A standardized region of interest of $9.72 \mathrm{~mm}^{3}$ was defined, including the first molar, the anterior half of the second molar and extending medially (towards the center of the palate) approximately $1 \mathrm{~mm}$ from the most palatal aspect of the crown of the first molar. All image reconstruction, reorientation and analysis of bone volume/total volume (BV/TV fraction) in the region of interest was performed using the software package of the scanning equipment software. 


\subsection{Histopathological Analysis}

The material, molars with the surrounding gingival tissues, was rinsed in saline and fixed in $10 \%$ formaldehyde solution for up to 7 days and then subjected to a process of demineralization, due to the presence of bone elements and teeth, for 15 days in a $18 \%$ solution of disodium ethylenediaminetetraacetate (EDTA). After the completion of decalcination, the material was cut into thin parts in the mesial-distal direction and treated by conventional histological procedures to paraffin molds. The final preparations were $4 \mu \mathrm{m}$ thick stained with hematoxylin and eosin (H\&E).

\subsection{Osteoclast Count}

Osteoclasts were identified by their morphology, location and positivity for the expression of tartrate-resistant acid phosphatase (TRAP), assessed by immunohistochemistry. Sections mounted on silanized slides were deparaffinized and rehydrated in a decreasing concentration of ethanol. Endogenous peroxidase was blocked with $3 \%$ hydrogen peroxide for $30 \mathrm{~min}$. To inhibit non-specific interactions of the primary antibody, the sections were incubated with a $5 \%$ skim milk blocking solution. Slides were washed in distilled water and incubated overnight at $4^{\circ} \mathrm{C}$ with goat polyclonal antibody for TRAP (cat\# sc-30833, Santa Cruz Biotechnology) diluted 1:100 in PBS containing 2\% BSA. Controls included omission of the primary antibody and also incubation with the same concentration of irrelevant IgG raised in goat. After washings in distilled water, the sections were incubated with biotinylated anti-goat antibodies for $30 \mathrm{~min}$, washed, incubated with streptavidin conjugated to HRP and finally with the DAB substrate, according to the protocol of the supplier of the visualization system. Subsequently, the sections were counterstained with Mayeŕs Hematoxylin and coverslips mounted. Osteoclasts were identified as large, multinucleated (3 or more visible nuclei) and TRAP-positive cells, located in the vicinity of the alveolar bone, from the apical region of the palatal root of the first molar to a $400 \mu \mathrm{m}$ distance from the palatal alveolar bone crest towards the midline (center of the palate). This analysis was performed in 4 semi-serial sections (spaced $100 \mu \mathrm{m}$ in the antero-posterior plane) from each specimen (20 sections for each experimental condition).

\subsection{Apoptosis Analysis}

Detection of active (cleaved) caspase- 3 in the cells of the gingival tissues was performed by immunohistochemistry. The same general protocol described for detection of TRAP was used, with the inclusion of an antigen retrieval step, performed in citrate buffer $\left(\mathrm{pH} \mathrm{6.0)}\right.$ at $95^{\circ} \mathrm{C}$ for $20 \mathrm{~min}$. After blocking of endogenous peroxidase and non-specific interactions of the primary antibody, the sections were washed in distilled water and incubated overnight at $4 \mathrm{C}$ with 1:50 dilution (in PBS containing 2\% BSA) of rabbit polyclonal antibodies against rat active caspase-3 (cat\# ab2302, Abcam). A total of 15 semi-serial sections (3 from 
each specimen from 5 different animals in each experimental condition) were assessed and the number of positively-stained cells counted in the area of interest (described in stereometric analysis) by a trained examiner who was unaware of the experimental condition of the sections.

\subsection{Measurements of the Factors of Inflammation}

The levels of the factors of inflammation, TNF- $\alpha$, IL- $1 \beta$, and IL-6, were determined using supernatants of $5 \%$ homogenate made up of fresh gingival tissues with deionized water. The measurement of cytokines was performed by enzyme-linked immunosorbent assay (ELISA) kits specific for rats in accordance with the instructions provided by the manufacturer (Invitrogen, Life Technologies, USA). Briefly, $50 \mu \mathrm{L}$ of sample was added to respective wells of a plate followed by $50 \mu \mathrm{L}$ of a standard diluent buffer, and $50 \mu \mathrm{L}$ of incubation buffer for IL- 6 assay. After incubation and washing of the wells $100 \mu \mathrm{L}$ of appropriate biotin conjugate was added, then the plates were left to incubate again followed by washing. Next stage included adding of $100 \mu \mathrm{L}$ and incubation with streptavidin-HRP working solution. After this step and washing, the plates were incubated with $100 \mu \mathrm{L}$ of stabilized chromogen followed by final adding of $100 \mu \mathrm{L}$ of stop solution. Absorbance of each well was read at $450 \mathrm{~nm}$ on ELISA microplate reader (Multiskan Ascent No354, Thermo Labsystems, Finland). A value of the sample concentration was calculated from a standard curve and twice multiplied. The results are presented as $\mathrm{pg}$ of the cytokines in $\mathrm{mg}$ of fresh gingival tissue.

\subsection{Statistical Analysis}

Data obtained from each experiment were analyzed using GraphPad Prism 5.0 (GraphPad Software Inc., San Diego, CA, USA). The objective of the analysis was to compare the results of each outcome according to the different tested compounds. The Shapiro-Wilk test was used to verify that the data had a normal distribution. Pairwise comparisons were performed by unpaired Student t-tests with Welch's correction for unequal variances and by one-way analysis of variance (ANOVA) followed by Tukey test for post-hoc pairwise comparisons. We adopted $5 \%$ as significant level $(\mathrm{p}<0.05)$.

\section{Results}

\subsection{Effects of Antifect on Histopathological Characteristics of Periodontitis in Rats}

Morphological changes in HE preparations are not visible in the Antifect treated group compare to the control. Figure 1(a) displays a cross section of rat molar with preserved structure of the tooth pulp tissue and periodontal ligament with a slight increase in the number of neutrophils. There are no perceptible changes in the alveolar bone. The histological characteristics of the HE slides of the experimental group I and II correspond completely to healthy tissue properties 
(Figure 1(b) and Figure 1(c)). Compared to the control animals (I and II group) the histopathology of the animals subjected to periodontitis receiving the vehicle (III group) displays notable changes both in soft tissue and hard tissue. The changes are characterized by the expansion of blood vessels in the periodontal tissue and gingival increase in the number of neutrophils and lymphocytes. Inflammation is followed by resorption of alveolar bone with the visible osteoclasts on the surface of bone and increased bone marrow space, strong deepening of the gingival sulcus and its separation from the cement teeth in the direction of the tooth root. The presence of red blood cells out of blood vessels indicating the bleeding (Figure 1(d)) is also evident. In the experimental groups IV and V (Figure 1(d) and Figure 1(e)), which were subjected to Antifect and ASA, it can be observed much smaller number of inflammatory cells in regard to the third group of rats. In periodontal and gingival tissue a larger number of fibroblasts persist compared to other groups of experimental animals, while the gingival sulcus is in the normal position.

\subsection{Oral Administration of Antifect Significantly Reduces Alveolar Bone Loss and Osteoclastogenesis Associated with LPS Induced Periodontal Disease}

Microcomputer tomography analysis indicates that attenuated inflammatory bone resorption in antifect treatment group (Figure 2). Group I and II had no
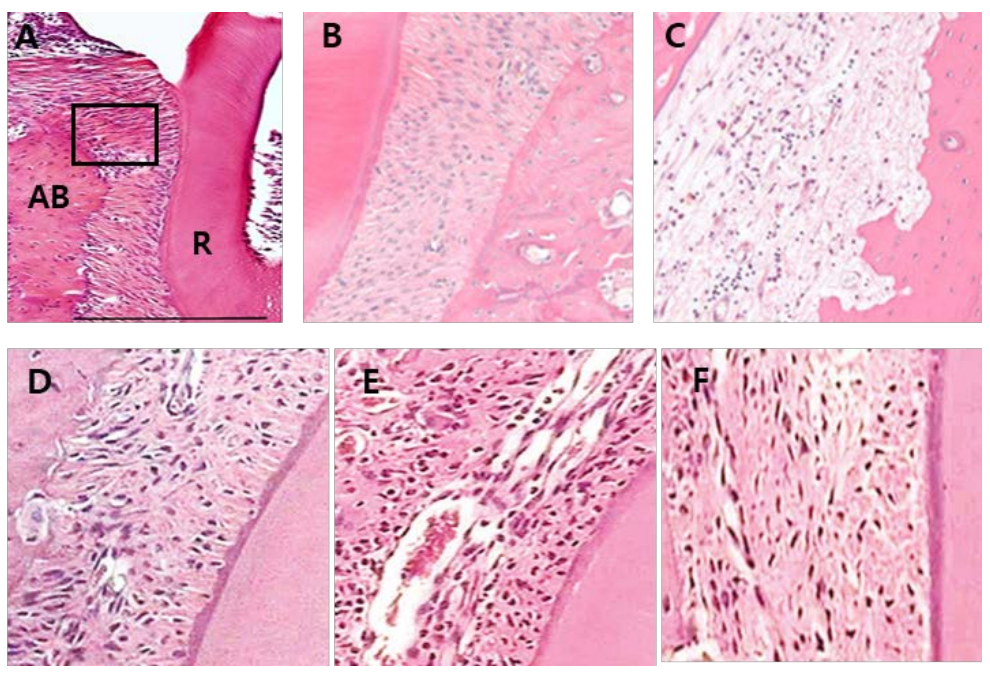

Figure 1. Histological characteristics of $\mathrm{H} / \mathrm{E}$-stained gingival tissues subjected to LPS-induced periodontal disease according to the experimental groups. (a) Palatal region where the injections of LPS were performed, corresponding to the rectangular box. (b) The contralateral side (control) was injected with the same volume of vehicle (PBS) in the same region. (c) Intense inflammatory in gingival tissues produced by LPS injections. (d) Antifect treated animals (group IV) in comparison to (e) LPS-vehicle control (group III), (f) ASA treated animals (group V). Images were obtained at $200 \times$ magnification. High power magnification $(600 \times)$ images detail the morphological characteristics of mononuclear and PMNs cell as they were considered for differential counting. The area of interest islocated at $400 \mu \mathrm{m}$ of the tooth, corresponding to the site of injections and the bulk of inflammatory infiltrate. $\mathrm{AB}$, alveolar bone. $\mathrm{R}$, root. 

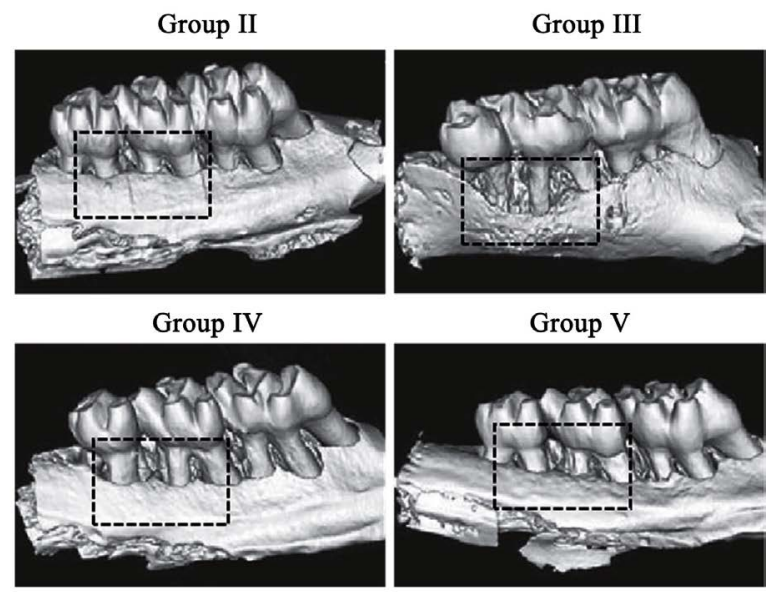

Group V
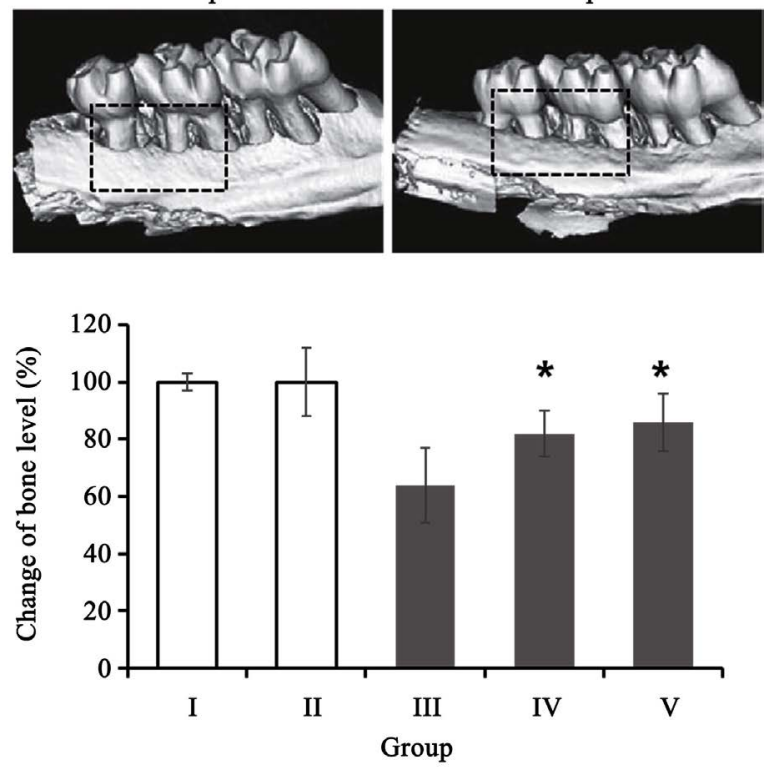

Figure 2. Effects of antifect and ASA on alveolar bone loss in periodontitis model. Palatal view of representative images of three dimensional reconstructions of the hemi-maxillaein each experimental condition. The bar graph presents the change in bone volume fraction (BVF) in comparison to control (100\%) in the standardized region of interest, according to the experimental condition. Data from hemi-maxillae of each experimental condition analyzed by ANOVA followed by Tukey post-hoc test. $\left(^{*}\right)$ indicates significant difference $(\mathrm{p}<0.05)$ in comparison to positive control group.

effect, and specimens from the LPS non treated animals were not scanned because previous Figure 1 shows that this vehicle does not affect the extent of inflammatory bone resorption in this model. The main purpose of this study was to compare the effects of Antifect and vehicle, ASA on alveolar bone resorption associated with the LPS-induced model of periodontal disease in an experiment performed concomitantly, took a $\mu \mathrm{CT}$ images and compare each group. With same histological analysis, alveolar bone level and bony defected area significantly increase in group III. However, with 15 days of compound treatment, increase of alveolar bone level and decrease of defected area also observed in group IV and V (Figure 2(a)). Long-term ASA administration reported recovery of inflammatory symptom of periodontitis patient [24], but gastric-intestinal irritation limits its long-term treatment. Antifect treatment group (IV) consistently showed decrease of alveolar bone loss similar with ASA treatment group (V) (Figure 2(b)).

Of primary importance, the number of TRAP-positive multinucleated cells, 
identified by immunohistochemistry, was significantly decreased only in the group IV even not in the group V, which supports the interpretation that the bone-sparing effects of this compound observed in the $\mu \mathrm{CT}$ analysis are due, at least in part, to the inhibition of osteoclastogenesis in vivo. ASA treatment had no effect on the number of osteoclasts present in the periodontal and gingival tissue (Figure 3).

\subsection{Decrease of Apoptosis in the Gingival Tissues}

Increased apoptosis in inflammation may generate a loop of sustained activation of the immune response, as dying cells release damage-associated molecular patterns (DAMPs). Inflammation induced by LPS injections increased apoptosis in the periodontal microenvironment, as indicated by significant increases of active caspase-3-stained cells in the gingival tissues. This increase in apoptosis was statistically significant in the alveolar bone crest (osteocytes) in all LPS-treated group. And its distribution focused in the bony defected area and alveolar crest area which was most activated inflammatory area. Administration of Antifect, but not of ASA treatment group, potently and significantly reduced the number of apoptotic cells in the gingival tissues and also of osteocytes in the alveolar bone crest, both in the presence and absence of LPS-induced inflammation (Figure 4).

\subsection{Effects of Antifect on the Gingival Concentrations of TNF- $\alpha$, IL- $1 \beta$ and IL- 6 of Rats}

The treatment with Antifect and ASA (IV and V group) significantly decreased the level of TNF- $\alpha$, IL- 6 and IL- $1 \beta$ in the gingival tissue with LPS-induced periodontitis when compared to the untreated group (III). There was no statistically significant difference of the levels of cytokines between groups which did not receive LPS (I and II). ASA treated group (V group) significantly reduced the levels of IL- $1 \beta$, but not IL- 6 and TNF- $\alpha$, in comparison to the group that treated antifect (IV) (Figure 5).

\section{Discussion}

Wistar rats were chosen to be used in the study as a model for periodontal disease since it has been shown that periodontal anatomy of molar region in rats are similar to that in humans. It has been proven that both, topical application, as well as the injection of LPS in mouse or rat periodontal tissue and gingiva develops common inflammatory changes, such as junctional epithelial disruption, infiltration of leukocytes and edema of the subepithelial connective tissue due to inflammatory cell infiltration. Additionally, LPS injection in rats periodontal tissue and gingiva shows significant alveolar bone loss starting at second day after periodontitis induction, reaching a top at seventh day [23]. Our histopathological findings in periodontal tissue and gingiva, obtained from the third group of experimental animals, show similar changes in these tissues. Intragingival 

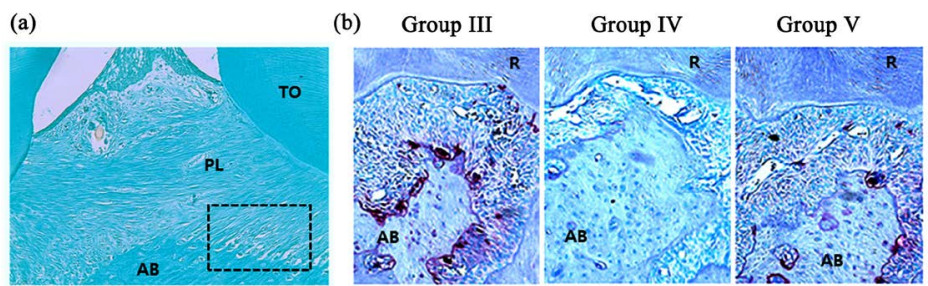

(c)

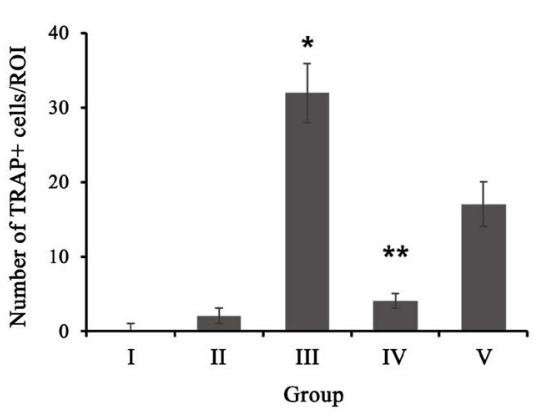

Figure 3. Effects of antifect and ASA on osteoclast differentiation. (a) Immunolocalization of TRAP-positive cells with three or more nuclei (arrows) close to the surface of alveolar bone tissue. Detail of osteoclast identified by the morphology, location and positivity for TRAP expression by immunohistochemistry (50× magnification). Representative images were obtained at $50 \times$ magnification. TO, tooth. PL, periodontal ligament. R, root, $\mathrm{AB}$, alveolar bone. (b) Representative images of the experimental groups at $200 \times$ magnification: Group II (a), Group III (b), Group IV (c) and Group V (d) in the presence of LPS injections. (c) Corresponding Counting of osteoclasts in the defined standardized area of interest. $\left.{ }^{*}\right)$ indicates significant difference $\mathrm{p}<0.05$ in comparison to negative control group. Bars indicate means and vertical lines standard error of mean of four semi-serial sections from each of five different animals in each experimental group.

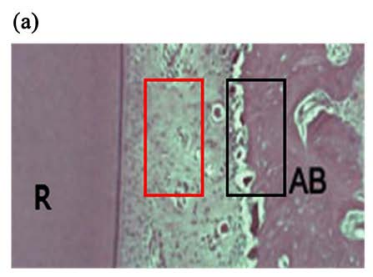

(c)

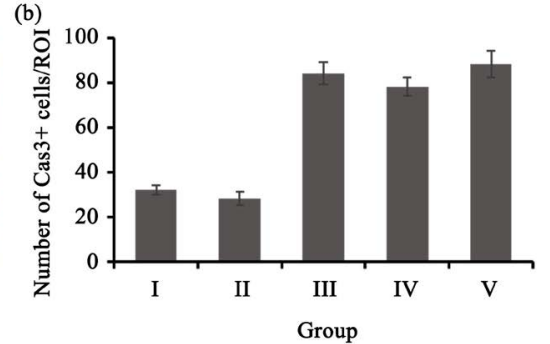

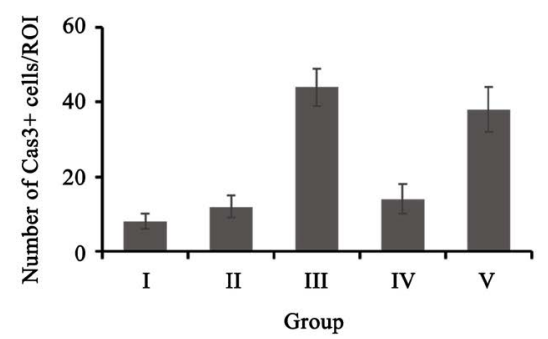

Figure 4. Effects of Antifect and ASA on apoptosis. (a) Areas in which the number of caspase- 3 positive cells were counted in the gingival tissue (red square) and bone crest (black square). Image was obtained at $50 \times$ magnification. $\mathrm{R}$, root. $\mathrm{AB}$, alveolar bone. (b) Number of caspase- 3 positive cells in the gingival tissues. (c) Number of caspase- 3 positive cells on bone crest. $\left({ }^{*}\right)$ indicates significant difference $(p<0.05)$ in comparison to negative control group. Bars indicate means and vertical lines standard error of mean of at the four semi-serial sections from each of five different animals in each experimental group. 

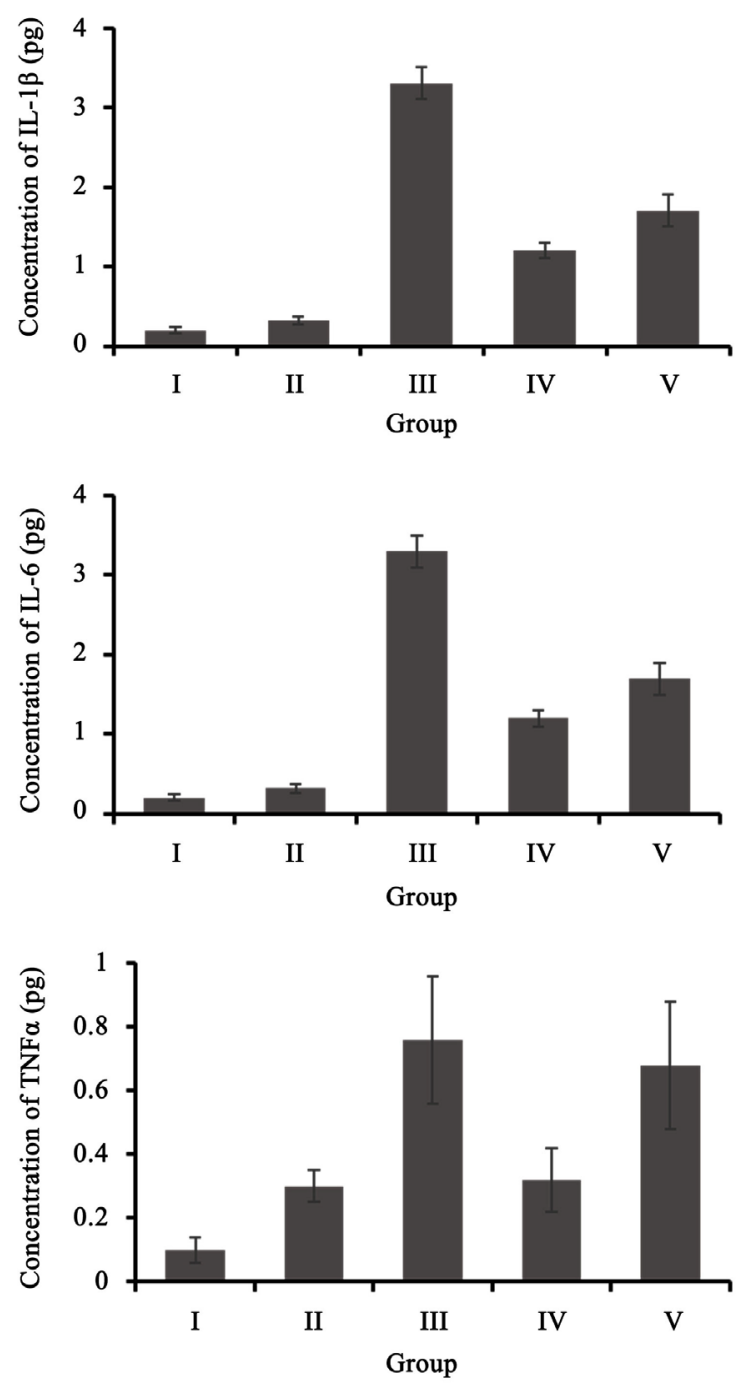

Figure 5. The levels of the cytokines IL-1 $\beta$ (A), IL-6 (B) and TNF- $\alpha$ (C) in the gingival tissues of the rats. Rats from I group were injected with saline and treated with distilledwater; II group injected with saline, treated with antifect; III group injected with LPS, treated with vehicle; IV group injected with LPS, treated with the antifect; V group injected with LPS, treated with ASA. The extract significantly decreased the levels of the cytokines (IV and V group) compared to untreated group of animals (III). Values represent means \pm standard deviation $(\mathrm{N}=6)$. Bars indicate the mean values and vertical lines standard deviations. Different letters in the columns show significant differences among varieties $(\mathrm{p}<0.05)$.

injection of $1 \mu \mathrm{L}$ of LPS solution, twice for ten days, in interdental papilla between the first and second molars induced inflammatory responses which implied drastic changes in histological appearance, activation of immune cells and producing of proinflammatory cytokines in periodontal tissue (III group).

Having in mind that periodontitis is a very common condition in humans, there is an ongoing search of adequate preventive or therapeutic procedures. It has been shown that some substances, such as pharmacologic COX-2 inhibitor such as acetylsalicylic acid (commercially "aspirin"), have protective effects on 
the periodontal tissue and alveolar bone, when administered with LPS. It is believed that this effect is the result of immunomodulatory properties of ASA due to their ability to inhibit the inflammatory signaling pathway composed COX-2 and prostaglandin synthesis, also, osteoclastic bone resorption and osteoclast formation in the presence of RANKL (receptor activator of nuclear factor kappa-B ligand) mediated by inhibiting of prostaglandin synthesis [25]. Interestingly, ASA and antifect treatment both present similar antiinflammatory effects, which may be derived from their MMP-inhibiting properties. However, these compounds may have distinct bone-sparing properties, as the decrease in the apoptosis of osteocytes observed only with administration of antifect may also be bone-protective since apoptotic osteocytes are associated with increased bone resorption and secretion of RANKL. These results reflect inhibiting effect of osteoclastogenesis of antifect may be associated with other candidate signaling such as antioxidative signaling.

The control groups (I and II) were injected with saline solution, instead of LPS, in order to investigate possible effects of the injection on the initiation of inflammation and bone destruction. The histopathology of the periodontal of group I, which corresponded to the healthy tissue, showed similar appearance to the group II, as it was also shown by immunoassay where there was no statistically difference in the level of cytokines between these groups. But, bacterial stimulation, especially by LPS, has been described to increase the secretion of proinflammatory cytokines by gingival inflammatory cells, especially key cytokines such as TNF- $\alpha$, IL- $1 \beta$ and IL-6 [26]. These cytokines play a relevant role in the initiation and development of periodontitis amplifying the immune response and activating of immune and nonimmune cells [27]. They are also able to provoke remarkable tissue damage by induction of collagenase in fibroblasts and osteoclasts activation [28]. The synergism between IL- $1 \beta$ and TNF- $\alpha$ and between IL- 6 and TNF- $\alpha$ have been found, whereby TNF- $\alpha$ is likely more important cytokine. It has also been noticed that healthy gingival tissue may contain inflammatory cytokines including the ones mentioned above present in low amounts [29]. This implies that cytokines are actually considerable factors for the maintenance of normal tissue homeostasis. Previous report about anti-allergic effects of Antifect was related to activity of its polyphenols which conspicuously reduced inflammatory process in mice induced by tryptanthrin and histamine [30]. Our study has shown that Antifect manifests anti-inflammatory effects in rats by significantly decreasing the levels of pro-inflammatory cytokines IL- $1 \beta$, IL-6 and TNF- $\alpha$ (Figure 5) with visible histological changes. It is evident that the administration of the extract in IV and V groups of rats modulated host response with extensive reduction of inflammatory infiltrate in comparison to III group. Immunoassay revealed that there were not statistically significant differences in the levels of the factors of inflammation IL- 6 and TNF- $\alpha$ in IV and V groups of the rats. Because, the COX-2 inhibitory activity also reported in I. tinctoria extract [19]. Generally, there were no differ- 
ences in histopathological characteristics between these group IV and V. But in anti-apoptotic effect and inhibition of bone resorption significantly increased in group IV. Usually occur of tissue destruction and cell apoptosis under acute inflammatory condition due to ROS (reactive oxygen species) stress [9]. I. tinctoria extracts known as contained high antioxidative molecules such as polyphenol, indoles also. And antiviral [31], antimicrobial effects [32] of I. tinctoria also assist inhibitory effect of periodontal inflammatory tissue destruction.

\section{Conclusion}

This study demonstrated that the oral administration of the extract from I. tinctorial Antifect had a suppressive effect on the LPS-induced inflammation of the rats' gingival tissue. The decrease of bony resorption, tissue destruction and cytokines production IL- 6 , IL- $1 \beta$ and TNF- $\alpha$ was in correlation with the reduction of the inflammatory process confirmed by histopathological analysis. In general, the results suggest that Antifect could act as a potential therapeutic and complementary agent in the treatment of periodontal disease. However, further studies should be conducted in order to confirm and define the efficiency of humans in certain clinical researches.

\section{Conflicts of Interest}

The authors declare no conflicts of interest regarding the publication of this paper.

\section{References}

[1] Kolenbrander, P.E. (1993) Coaggregation of Human Oral Bacteria: Potential Role in the Accretion of Dental Plaque. Journal of Applied Bacteriology, 74, 79S-86S. https://doi.org/10.1111/j.1365-2672.1993.tb04344.x

[2] Oliver, D.R. (1968) The Etiology of Periodontal Disease. The Journal of the Missouri Dental Association, 48, 12-18.

[3] Lang, N.P. (2014) Commentary: Bacteria Play a Critical Role in the Etiology of Periodontal Disease. Journal of Periodontology, 85, 211-213. https://doi.org/10.1902/jop.2013.130699

[4] Golz, L., et al. (2014) LPS from P. gingivalis and Hypoxia Increases Oxidative Stress in Periodontal Ligament Fibroblasts and Contributes to Periodontitis. Mediators of Inflammation, 2014, Article ID: 986264. https://doi.org/10.1155/2014/986264

[5] Stein, S.H., Borke, J.L. and Cummings, L.A. (1999) Effects of Chronic Adult Periodontitis and Endotoxin (LPS) on Gingival Fibroblast Plasma Membrane Ca -Pump. Connective Tissue Research, 40, 59-66. https://doi.org/10.3109/03008209909005278

[6] Sharma, N., et al. (2014) Cytokine Gene Polymorphism (Interleukin-1Beta +3954, Interleukin-6 [-597/-174] and Tumor Necrosis Factor-Alpha -308) in Chronic Periodontitis with and without Type 2 Diabetes Mellitus. Indian Journal of Dental Research, 25, 375-380. https://doi.org/10.4103/0970-9290.138343

[7] Teles, F.R., Teles, R.P., Martin, L., Socransky, S.S. and Haffajee, A.D. (2012) Relationships among Interleukin-6, Tumor Necrosis Factor-Alpha, Adipokines, Vitamin 
D, and Chronic Periodontitis. Journal of Periodontology, 83, 1183-1191. https://doi.org/10.1902/jop.2011.110346

[8] Babel, N., et al. (2006) Analysis of Tumor Necrosis Factor-Alpha, Transforming Growth Factor-Beta, Interleukin-10, IL-6, and Interferon-Gamma Gene Polymorphisms in Patients with Chronic Periodontitis. Journal of Periodontology, 77, 1978-1983. https://doi.org/10.1902/jop.2006.050315

[9] Kanzaki, H., et al. (2017) Pathways that Regulate ROS Scavenging Enzymes, and Their Role in Defense against Tissue Destruction in Periodontitis. Frontiers in Physiology, 8, 351. https://doi.org/10.3389/fphys.2017.00351

[10] Chiang, C.Y., et al. (2016) A Salvia miltiorrhiza Ethanol Extract Ameliorates Tissue Destruction Caused by Experimental Periodontitis in Rats. Journal of Periodontal Research, 51, 133-139. https://doi.org/10.1111/jre.12292

[11] Yoshinaga, Y., et al. (2014) Green Tea Extract Inhibits the Onset of Periodontal Destruction in Rat Experimental Periodontitis. Journal of Periodontal Research, 49, 652-659. https://doi.org/10.1111/jre.12147

[12] Barrella, G.E., et al. (2012) Evaluation of the Effect of an Organic Extract Obtained from Ipomoea alba L. on Experimental Periodontitis in Rats. Brazilian Oral Research, 26, 158-164. https://doi.org/10.1590/S1806-83242012000200012

[13] Anand, P., Kunnumakkara, A.B., Newman, R.A. and Aggarwal, B.B. (2007) Bioavailability of Curcumin: Problems and Promises. Molecular Pharmacology, 4, 807-818. https://doi.org/10.1021/mp700113r

[14] Liou, C.J., Huang, W.C., Kuo, M.L., Yang, R.C. and Shen, J.J. (2010) Long-Term Oral Administration of Gynostemma pentaphyllum Extract Attenuates Airway Inflammation and Th2 Cell Activities in Ovalbumin-Sensitized Mice. Food and Chemical Toxicology, 48, 2592-2598. https://doi.org/10.1016/j.fct.2010.06.020

[15] Kerdar, T., Rabienejad, N., Alikhani, Y., Moradkhani, S. and Dastan, D. (2019) Clinical, in Vitro and Phytochemical, Studies of Scrophularia striata Mouthwash on Chronic Periodontitis Disease. Journal of Ethnopharmacology, 239, 111872. https://doi.org/10.1016/j.jep.2019.111872

[16] Heinemann, C., Schliemann-Willers, S., Oberthür, C., Hamburger, M. and Elsner, P. (2004) Prevention of Experimentally Induced Irritant Contact Dermatitis by Extracts of Isatis tinctoria Compared to Pure Tryptanthrin and Its Impact on UVB-Induced Erythema. Planta Medica, 70, 385-390. https://doi.org/10.1055/s-2004-818963

[17] Danz, H., Baumann, D. and Hamburger, M. (2002) Quantitative Determination of the Dual COX-2/5-LOX Inhibitor Tryptanthrin in Isatis tinctoria by ESI-LC-MS. Planta Medica, 68, 152-157. https://doi.org/10.1055/s-2002-20252

[18] Seifert, K. and Unger, W. (1994) Insecticidal and Fungicidal Compounds from Isatis tinctoria. Zeitschrift für Naturforschung C, 49, 44-48. https://doi.org/10.1515/znc-1994-1-208

[19] Danz, H., Stoyanova, S., Wippich, P., Brattström, A. and Hamburger, M. (2001) Identification and Isolation of the Cyclooxygenase-2 Inhibitory Principle in Isatis tinctoria. Planta Medica, 67, 411-416. https://doi.org/10.1055/s-2001-15805

[20] Zhang, D., et al. (2019) Indole Alkaloid Glycosides from Isatis tinctoria Roots. Natural Product Research, 1-7. https://doi.org/10.1080/14786419.2019.1624960

[21] Zhou, J. and Qu, F. (2011) Analysis of the Extracts of Isatis tinctoria by New Analytical Approaches of HPLC, MS and NMR. African Journal of Traditional, Complementary and Alternative Medicines, 8, 33-45. 
https://doi.org/10.4314/ajtcam.v8i5S.13

[22] Brattstrom, A., et al. (2010) The Plant Extract Isatis tinctoria L. Extract (ITE) Inhibits Allergen-Induced Airway Inflammation and Hyperreactivity in Mice. Phytomedicine, 17, 551-556. https://doi.org/10.1016/j.phymed.2009.11.003

[23] Dumitrescu, A.L., Abd El-Aleem, S., Morales-Aza, B. and Donaldson, L.F. (2004) A Model of Periodontitis in the Rat: Effect of Lipopolysaccharide on Bone Resorption, Osteoclast Activity, and Local Peptidergic Innervation. Journal of Clinical Periodontology, 31, 596-603. https://doi.org/10.1111/j.1600-051X.2004.00528.x

[24] Zhang, Z.S. (2018) Association between COX2 -765G/C Polymorphism and Periodontitis in Chinese Population: A Meta-Analysis. BMC Oral Health, 18, 32. https://doi.org/10.1186/s12903-018-0483-9

[25] Kamon, M., Fujita, D., Goto, N., Amano, H. and Sakamoto, K. (2008) Prostaglandin $\mathrm{F}_{2 \alpha}$ Negatively Regulates Bone Resorption in Murine Osteoclast Development. Prostaglandins \& Other Lipid Mediators, 87, 26-33. https://doi.org/10.1016/j.prostaglandins.2008.06.002

[26] Acharya, A.B., Thakur, S., Muddapur, M.V. and Kulkarni, R.D. (2016) Tumor Necrosis Factor-Alpha, Interleukin- 4 and -6 in the Serum of Health, Chronic Periodontitis, and Type 2 Diabetes Mellitus. Journal of Indian Society of Periodontology, 20, 509-513. https://doi.org/10.4103/0972-124X.201694

[27] Martin, P.L., et al. (2005) Reviews Preclinical Safety and Immune-Modulating Effects of Therapeutic Monoclonal Antibodies to Interleukin-6 and Tumor Necrosis Factor-alpha in Cynomolgus Macaques. Journal of Immunotoxicology, 1, 131-139. https://doi.org/10.1080/15476910490894904

[28] Fujikawa, Y., Sabokbar, A., Neale, S.D., Itonaga, I., Torisu, T. and Athanasou, N.A. (2001) The Effect of Macrophage-Colony Stimulating Factor and Other Humoral Factors (Interleukin-1, -3, -6, and -11, Tumor Necrosis Factor-Alpha, and Granulocyte Macrophage-Colony Stimulating Factor) on Human Osteoclast Formation from Circulating Cells. Bone, 28, 261-267. https://doi.org/10.1016/S8756-3282(00)00453-1

[29] Stashenko, P., Jandinski, J.J., Fujiyoshi, P., Rynar, J. and Socransky, S.S. (1991) Tissue Levels of Bone Resorptive Cytokines in Periodontal Disease. Journal of Periodontology, 62, 504-509. https://doi.org/10.1902/jop.1991.62.8.504

[30] Recio, M.C., Cerdá-Nicolás, M., Potterat, O., Hamburger, M. and Ríos, J.-L. (2006) Anti-Inflammatory and Antiallergic Activity in Vivo of Lipophilic Isatis tinctoria Extracts and Tryptanthrin. Planta Medica, 72, 539-546. https://doi.org/10.1055/s-2006-931562

[31] Gao, B., Zhang, J. and Xie, L. (2018) Structure Analysis of Effective Chemical Compounds against Dengue Viruses Isolated from Isatis tinctoria. Canadian Journal of Infectious Diseases and Medical Microbiology, 2018, Article ID: 3217473. https://doi.org/10.1155/2018/3217473

[32] Ahmad, I. and Fatima, I. (2008) Butyrylcholinesterase, Lipoxygenase Inhibiting and Antifungal Alkaloids from Isatis tinctoria. Journal of Enzyme Inhibition and Medicinal Chemistry, 23, 313-316. https://doi.org/10.1080/14756360701536455 